\title{
Study the Visual Pathway in Patients Taking Hydroxychloroquine
}

\author{
Mandijo Nukatij* \\ Department of Ophthalmology and optic study, University in Wuhan, China
}

*Corresponding author: Mandijo Nukatij, Department of Ophthalmology and Optic

Received Date: June 19, 2019

study, University in Wuhan, China.

Published Date: June 27, 2019

\begin{abstract}
Hydroxychloroquine is considered a disease-modifying anti-rheumatic drug (DMARD). It can decrease the pain and swelling of arthritis. It may prevent joint damage and reduce the risk of long-term disability. This drug is having certain side effect on retina in visual system. The purpose of this study is to check the visual pathway of this patients using visual evoked potential (VEP). Twenty-eight patients suffering from rheumatic arthritis were selected in this study. They were under $200 \mathrm{mg} /$ day of HCQ treatment for at least 19 months. VEP test was examined in all patients. The results obtained was compared with the control group. The mean latency and amplitude of VEP in patient group were 88/8.26 msec and 9.7/3.15 $\mu \mathrm{v}$ and 97/9.31 msec and 8.4/7.18 $\mu \mathrm{v}$ in control group respectively. There were not statistical differences between mean latency and amplitude of VEP, P100 peak as far as case and control groups are concerned. It can be concluded that Hydroxychloroquine does not affect visual pathway seriously.
\end{abstract}

Keywords: Hydroxychloroquine; Visual pathway; Visual evoked potential; Rheumatoid arthritis

\section{Introduction}

Hydroxychloroquine is in a class of medications that was first used to prevent and treat malaria. Nowadays, it is used to treat rheumatoid arthritis, some symptoms of lupus, childhood arthritis and other autoimmune diseases. It is not clear why hydroxychloroquine is effective at treating autoimmune diseases. Rheumatoid Arthritis is a chronic inflammatory disorder that can affect joints. Taking Hydroxychloroquine for long term or at high doses may cause irreversible damage to different organs of body. Some of the common side effect of this drug include headache, dizziness, ringing in your ears, nausea, vomiting, stomach pain, loss of appetite, weight loss, mood changes, skin rash or itching. It is also reported that swelling or color changes in the eyes may occur after using this drug. It is important to diagnose the effect of the drug on retina before the patient have those symptoms. In this regard, recommend actions for screening hydroxychloroquine retinopathy have recently been changed by American Academy of Ophthalmology taking in to account new published data on toxicity prevalence, risk factors, location of onset in retina and the efficacy of screening test. A baseline examination should be performed at the initiation of treatment to rule out pre-existing retinopathy. The screening test such as Visual Evoked Potential (VEP) and Electroretinogram (ERG) should be performed every year from the fifth year of treatment. Visual Evoked Potential (VEP) is used to measure the functional integrity of the visual pathways from retina via the optic nerves to the visual cortex of the brain. Recording of VEP is an important means of obtaining reproducible and quantitative data of function of the anterior visual pathways [1]. A research was made to study retinal changes in multiple sclerosis patients with abnormal VEP. The result shows retinal dysfunction of MS patients which is proved by increase in latency of ERG, b wave [2]. Another study was done on visual pathway disturbances of photophobia patients using VEP techniques, concluded that pattern reversal checkerboard in VEP is more efficient for this diagnosis rather than flash one [3]. A case report was about a patient complaining from seeing colored rings around the light after refractive surgery with taking Amiodarone. All screening tests for visual pathway (VEP, ERG and EOG) were abnormal. The symptom improved after asking the patient to stop using the medication [4]. In 2014, another research was accomplished on effect of Depakine in epileptic patients using ERG [5]. Based on the researches was mentioned and other studies, it shows that VEP and ERG are reliable test for checking the toxicity of medications in visual pathway.

\section{Material and Methods}

In this case control study, twenty-six patients with Rheumatoid Arthritis and history of taking Hydroxychloroquine as treatment 
for at least 19 months and the same number healthy subjects were selected as case and control group respectively. Visual Evoked Potential (VEP) was tested in both groups. Three electrodes active, reference and earth were used to attach to occipital, vertex and forehead respectively. Latency and amplitude parameters of VEP, P100 was measured for each subject. Case group had 20 females and 6 males. Control group included 18 females and 8 males; their visual acuity was approximately similar to case group.

\section{Result}

The mean latency of VEP, P100 peak/ S.D in case and control group were 88/8.26 and 97/9.31 (msec) and the mean amplitudes were $9.7 / 3.15$ and 8.4/7.18 $\mu \mathrm{v}$ respectively which shows the differences between mean latency and amplitude of VEP, P100 peak were not statistically significant.

\section{Discussion}

The result of present work shows no significant statistically differences between VEP parameters i.e. amplitude and latency of VEP, P100 peak in case and control groups. VEP is a common technique which is used by neurologist and ophthalmologist to check the visual pathway based on electrical information recorded from the visual cortex in response to stimulation of retina. Considering above. The result of a study was done in patients taking hydroxychloroquine, using contrast sensitivity test (CST), electroretinography (ERG) and pattern reversal visual evoked potential (PVEP) showed that CST can be the most sensitive of other tests in patients [6]. A study was done by Cavagna et al. indicated that electroretinogram can be helpful for early detection of retinal alterations during hydroxychloroquine treatment whereas EOG and VEP are inefficient for early detection of hydroxychloroquine retinopathy [7]. There are other studies that show the integrity of visual pathway can be tested using this method [8]. Other work was done lately for study the effect of anti-epileptic drug on visual pathway using VEP [9]. It shows that screening visul pathway during treatment by different medication and in different diseases can be helpful for doctors to decide if using the drug affect the visual pathway or not and if it is necessary to stop taking the medication.

\section{Conclusion}

In our study, VEP was examined in Patients taking Hydroxychloroquine to see if using this medication after a while can cause possible changes in VEP parameters. According to the result, we could not find any significant difference in VEP latency and amplitude compared to case and control groups. Retinal disturbances may affect visual pathway and this effect can be tested by VEP. So, more study is needed to prove this result in patients using this drug.

\section{Acknowledgement}

None.

\section{Conflicts of Interest}

No conflict of interest.

\section{References}

1. Aminoff Michael J, Goodin Douglas S (1994) Visual Evoked Potentials. Journal of Clinical Neurophysiology 11(5).

2. SM Shushtarian, F Adhami Moghadam, M Naser (2017) Electroretinographic Changes in Multiple Sclerosis Patients with Abnormal Visual Evoked Potentials. Journal of Ophthalmic and Optometric Sciences 1(3): 34-38.

3. M Naser, SM Shushtarian, H Abdolhoseinpour, T Davari (2014) Selection of Suitable Visual Stimulator for Recording of Visual Evoked Potential in Photophobia Patients. Indian Journal of Applied Research 4(9).

4. M Naser, SM Shushtarian, A Shojaei, F Adlami-Moghdam (2017) Visual Disturbance in a Patient with Amiodarone Treatment Following Refractive Surgery. Journal of Ophthalmic and Optometric Sciences 1(3).

5. M Naser, SM Shushtarian (2014) Study the effect of depakine on retina of epileptic patients using electroretinogram. International Journal of Scientific Research 3 (1): 392-393.

6. Bishara SA, Matamoros N (1989) Evaluation of several tests in screening for chloroquine maculopathy. Eye 3: 777-782.

7. Cavagna L, Rossi P, Bogliolo L, Antoniazzi E, Gelmi C, et al. (2002) Early electroretinographic changes in elderly RA patients treated with hydroxychloroquine. Reumatismo 54(3): 226-231.

8. Weinstein GW, Odom JV, Cavender S (1991) Visually evoked potentials and electroretinography in neurologic evaluation. Neurol Clin 9(1): 225242.

9. Maryam Naser, Shoniz Asnovil (2019) Visual Evoked Potential in Patients with Valproic Acid Treatment. W J Opthalmol \& Vision Res. 1(5): 1-2. 